\title{
A NOTE ON EXHAUSTION OF HYPERBOLIC COMPLEX MANIFOLDS
}

\author{
NINH VAN THU ${ }^{1,2}$ AND TRINH HUY VU ${ }^{1}$
}

\begin{abstract}
The purpose of this article is to investigate a hyperbolic complex manifold $M$ exhausted by a pseudoconvex domain $\Omega$ in $\mathbb{C}^{n}$ via an exhausting sequence $\left\{f_{j}: \Omega \rightarrow\right.$ $M\}$ such that $f_{j}^{-1}(a)$ converges to a boundary point $\xi_{0} \in \partial \Omega$ for some point $a \in M$.
\end{abstract}

\section{INTRODUCTION}

Let $M$ and $\Omega$ be two complex manifolds. One says that $\Omega$ can exhaust $M$ or $M$ can be exhausted by $\Omega$ if for any compact subset $K$ of $M$ there is a holomorphic embedding $f_{K}: \Omega \rightarrow M$ such that $f_{K}(\Omega) \supset K$. In particular, one says that $M$ is a monotone union of $\Omega$ via a sequence of holomorphic embeddings $f_{j}: \Omega \rightarrow M$ if $f_{j}(\Omega) \subset f_{j+1}(\Omega)$ for all $j$ and $M=\bigcup_{j=1}^{\infty} f_{j}(\Omega)$ (see [FS77, Fr83]).

In [Fr86, Theorem 1], there exists a bounded domain $D$ in $\mathbb{C}^{n}$ such that $D$ can exhaust any domain in $\mathbb{C}^{n}$. In addition, the unit ball $\mathbb{B}^{n}$ in $\mathbb{C}^{n}$ can exhaust many complex manifods, which are not biholomorphically equivalent to each other (see [For04, FS77]). However, if $M$ in addition is hyperbolic then $M$ must be biholomorphically equivalent to $\mathbb{B}^{n}$ (cf. [FS77]). Furthermore, any $n$-dimensional hyperbolic complex manifold, exhausted by a homogeneous bounded domain $D$ in $\mathbb{C}^{n}$, is biholomorphically equivalent to $D$. As a consequence, although the polydisc $\mathbb{U}^{n}$ and the unit ball $\mathbb{B}^{n}$ are both homogeneous and there is a domain $U$ in $\mathbb{B}^{n}$ that contains almost all of $\mathbb{B}^{n}$, i.e., $\mathbb{B}^{n} \backslash U$ has measure zero (cf. [FS77, Theorem 1]) and is biholomorphically equivalent to $\mathbb{U}^{n}$, but $\mathbb{U}^{n}$ cannot exhaust the unit ball $\mathbb{B}^{n}$ since it is well-known that $\mathbb{U}^{n}$ is not biholomorphically equivalent to $\mathbb{B}^{n}$.

Let $M$ be a hyperbolic complex manifold exhausted by a bounded domain $\Omega \subset \mathbb{C}^{n}$ via an exhausting sequence $\left\{f_{j}: \Omega \rightarrow M\right\}$. Let us fix a point $a \in M$. Then, thanks to the boundedness of $\Omega$, without loss of generality we may assume that $f_{j}^{-1}(a) \rightarrow p \in \bar{\Omega}$ as $j \rightarrow \infty$. If $p \in \Omega$, then one always has $M$ is biholomorphically equivalent to $\Omega$ (cf. Lemma 2.3 in Section 2).

The purpose of this paper is to investigate such a complex manifold $M$ with $p \in \partial \Omega$. More precisely, our first main result is the following theorem.

Theorem 1.1. Let $M$ be an $(n+1)$-dimensional hyperbolic complex manifold and let $\Omega$ be a pseudoconvex domain in $\mathbb{C}^{n+1}$ with $C^{\infty}$-smooth boundary. Suppose that $M$ can be exhausted by $\Omega$ via an exhausting sequence $\left\{f_{j}: \Omega \rightarrow M\right\}$. If there exists a point $a \in M$ such that the sequence $f_{j}^{-1}(a)$ converges $\Lambda$-nontangentially to a h-extendible boundary point $\xi_{0} \in \partial \Omega$ (see Definition 2.2 in Section 2 for definitions of the $\Lambda$-nontangentially

Date: June 9, 2020.

2010 Mathematics Subject Classification. Primary 32H02; Secondary 32M05, 32F18.

Key words and phrases. Hyperbolic complex manifold, exhausting sequence, $h$-extendible domain. 
convergence and of the h-extendibility), then $M$ is biholomorphically equivalent to the associated model $M_{P}$ for $\Omega$ at $\xi_{0}$.

When $\xi_{0}$ is a strongly pseudoconvex boundary point, we do not need the condition that the sequence $f_{j}^{-1}(a)$ converges $\Lambda$-nontangentially to $\xi_{0}$ as $j \rightarrow \infty$. Moreover, in this circumstance, the model $M_{P}$ is in fact biholomorphically equivalent to $M_{|z|^{2}}$, which is biholomorphically equivalent to the unit ball $\mathbb{B}^{n}$. More precisely, our second main result is the following theorem.

Theorem 1.2. Let $M$ be an $(n+1)$-dimensional hyperbolic complex manifold and let $\Omega$ be a pseudoconvex domain in $\mathbb{C}^{n+1}$. Suppose that $\partial \Omega$ is $\mathcal{C}^{2}$-smooth boundary near a strongly pseudoconvex boundary point $\xi_{0} \in \partial \Omega$. Suppose also that $M$ can be exhausted by $\Omega$ via an exhausting sequence $\left\{f_{j}: \Omega \rightarrow M\right\}$. If there exists a point $a \in M$ such that the sequence $\eta_{j}:=f_{j}^{-1}(a)$ converges to $\xi_{0}$, then $M$ is biholomorphically equivalent to the unit ball $\mathbb{B}^{n+1}$.

Notice that Theorem [1.2 is a local version of [DZ19, Theorem 1.1] and [Fr83, Theorem I] (see Corollary 3.4 in Section 3). We note that their proofs are based on the boundary estimate of the Fridman invariant and of the squeezing function for strongly pseudoconvex domains. However, in order to prove Theorem 1.1 and Theorem 1.2, we shall use the scaling technique, achieved recently in [Ber06, DN09, NN19].

By applying Theorem 1.2 and Lemma 2.3, we also prove that if a hyperbolic complex manifold $M$ exhausted by a general ellipsoid $D_{P}$ (see Section 4 for the definition of $D_{P}$ ), then $M$ is either biholomorphically equivalent to $D_{P}$ or the unit ball $\mathbb{B}^{n}$ (cf. Proposition 4.1 in Section (4). In particular, when $D_{P}$ is an ellipsoid $E_{m}\left(m \in \mathbb{Z}_{\geqslant 1}\right)$, given by

$$
E_{m}=\left\{(z, w) \in \mathbb{C}^{2}:|w|^{2}+|z|^{2 m}<1\right\},
$$

in fact Proposition 4.1 is a generalization of [Liu18, Theorem 1].

The organization of this paper is as follows: In Section 2 we provide some results concerning the normality of a sequence of biholomorphisms and the $h$-extendibility. In Section 3, we give our proofs of Theorem 1.1 and Theorem 1.2. Finally, the proof of Proposition 4.1 will be introduced in Section 4 .

\section{The NORMALITY AND THE $h$-EXTENDIBILITY}

2.1. The normality of a sequence of biholomorphisms. First of all, we recall the following definition (see [GK87] or [DN09]).

Definition 2.1. Let $\left\{\Omega_{i}\right\}_{i=1}^{\infty}$ be a sequence of open sets in a complex manifold $M$ and $\Omega_{0}$ be an open set of $M$. The sequence $\left\{\Omega_{i}\right\}_{i=1}^{\infty}$ is said to converge to $\Omega_{0}$ (written $\lim \Omega_{i}=\Omega_{0}$ ) if and only if

(i) For any compact set $K \subset \Omega_{0}$, there is an $i_{0}=i_{0}(K)$ such that $i \geqslant i_{0}$ implies that $K \subset \Omega_{i}$; and

(ii) If $K$ is a compact set which is contained in $\Omega_{i}$ for all sufficiently large $i$, then $K \subset \Omega_{0}$.

Next, we recall the following proposition, which is a generalization of the theorem of H. Cartan (see [DN09, GK87, [DT04]).

Proposition 2.1. Let $\left\{A_{i}\right\}_{i=1}^{\infty}$ and $\left\{\Omega_{i}\right\}_{i=1}^{\infty}$ be sequences of domains in a complex manifold $M$ with $\lim A_{i}=A_{0}$ and $\lim \Omega_{i}=\Omega_{0}$ for some (uniquely determined) domains $A_{0}$, 
$\Omega_{0}$ in $M$. Suppose that $\left\{f_{i}: A_{i} \rightarrow \Omega_{i}\right\}$ is a sequence of biholomorphic maps. Suppose also that the sequence $\left\{f_{i}: A_{i} \rightarrow M\right\}$ converges uniformly on compact subsets of $A_{0}$ to a holomorphic map $F: A_{0} \rightarrow M$ and the sequence $\left\{g_{i}:=f_{i}^{-1}: \Omega_{i} \rightarrow M\right\}$ converges uniformly on compact subsets of $\Omega_{0}$ to a holomorphic map $G: \Omega_{0} \rightarrow M$. Then either of the following assertions holds.

(i) The sequence $\left\{f_{i}\right\}$ is compactly divergent, i.e., for each compact set $K \subset A_{0}$ and each compact set $L \subset \Omega_{0}$, there exists an integer $i_{0}$ such that $f_{i}(K) \cap L=\varnothing$ for $i \geqslant i_{0} ;$ or

(ii) There exists a subsequence $\left\{f_{i_{j}}\right\} \subset\left\{f_{i}\right\}$ such that the sequence $\left\{f_{i_{j}}\right\}$ converges uniformly on compact subsets of $A_{0}$ to a biholomorphic map $F: A_{0} \rightarrow \Omega_{0}$.

Remark 2.2. By [Ber94, Proposition 2.1] or [DN09, Proposition 2.2] and by the hypotheses of Theorem 1.1 and Theorem [1.2, it follows that for each compact subset $K \Subset M$ and each neighborhood $U$ of $\xi_{0}$ in $\mathbb{C}^{n+1}$, there exists an integer $j_{0}=j_{0}(K)$ such that $K \subset f_{j}(\Omega \cap U)$ for all $j \geqslant j_{0}$. Consequently, the sequence of domains $\left\{f_{j}(\Omega \cap U)\right\}$ converges to $M$.

We will finish this subsection by recalling the following lemma (cf. [Fr83, Lemma $1.1])$.

Lemma 2.3 (see [Fr83]). Let $M$ be a hyperbolic manifold of complex dimension $n$. Assume that $M$ can be exhausted by $\Omega$ via an exhausting sequence $\left\{f_{j}: \Omega \rightarrow M\right\}$, where $\Omega$ is a bounded domain in $\mathbb{C}^{n}$. Suppose that there is an interior point $a \in M$ such that $f_{j}^{-1}(a) \rightarrow p \in \Omega$. Then, $M$ is biholomorphically equivalent to $\Omega$.

2.2. The $h$-extendibility. In this subsection, we recall some definitions and notations given in Cat84, Yu95].

Let $\Omega$ be a smooth pseudoconvex domain in $\mathbb{C}^{n+1}$ and $p \in \partial \Omega$. Let $\rho$ be a local defining function for $\Omega$ near $p$. Suppose that the multitype $\mathcal{M}(p)=\left(1, m_{1}, \ldots, m_{n}\right)$ is finite. (See Cat84 for the notion of multitype.) Let us denote by $\Lambda=\left(1 / m_{1}, \ldots, 1 / m_{n}\right)$. Then, there are distinguished coordinates $(z, w)=\left(z_{1}, \ldots, z_{n}, w\right)$ such that $p=0$ and $\rho(z, w)$ can be expanded near 0 as follows:

$$
\rho(z, w)=\operatorname{Re}(w)+P(z)+R(z, w)
$$

where $P$ is a $\Lambda$-homogeneous plurisubharmonic polynomial that contains no pluriharmonic terms, $R$ is smooth and satisfies

$$
|R(z, w)| \leqslant C\left(|w|+\sum_{j=1}^{n}\left|z_{j}\right|^{m_{j}}\right)^{\gamma}
$$

for some constant $\gamma>1$ and $C>0$. Here and in what follows, a polynomial $P$ is called $\Lambda$-homogeneous if

$$
P\left(t^{1 / m_{1}} z_{1}, t^{1 / m_{2}} z_{2}, \ldots, t^{1 / m_{n}} z_{n}\right)=P(z), \forall t>0, \forall z \in \mathbb{C}^{n} .
$$

Definition 2.2 (see [NN19]). The domain $M_{P}=\left\{(z, w) \in \mathbb{C}^{n} \times \mathbb{C}: \operatorname{Re}(w)+P(z)<0\right\}$ is called an associated model of $\Omega$ at $p$. A boundary point $p \in \partial \Omega$ is called $h$-extendible if its associated model $M_{P}$ is $h$-extendible, i.e., $M_{P}$ is of finite type (see YYu94, Corollary 2.3]). In this circumstance, we say that a sequence $\left\{\eta_{j}=\left(\alpha_{j}, \beta_{j}\right)\right\} \subset \Omega$ converges 
$\Lambda$-nontangentially to $p$ if $\left|\operatorname{Im}\left(\beta_{j}\right)\right| \lesssim\left|\operatorname{dist}\left(\eta_{j}, \partial \Omega\right)\right|$ and $\sigma\left(\alpha_{j}\right) \lesssim\left|\operatorname{dist}\left(\eta_{j}, \partial \Omega\right)\right|$, where

$$
\sigma(z)=\sum_{k=1}^{n}\left|z_{k}\right|^{m_{k}}
$$

Throughout this paper, we use $\lesssim$ and $\gtrsim$ to denote inequalities up to a positive multiplicative constant. Moreover, we use $\approx$ for the combination of $\lesssim$ and $\gtrsim$. In addition, $\operatorname{dist}(z, \partial \Omega)$ denotes the Euclidean distance from $z$ to $\partial \Omega$. Furthermore, for $\mu>0$ we denote by $\mathcal{O}(\mu, \Lambda)$ the set of all smooth functions $f$ defined near the origin of $\mathbb{C}^{n}$ such that

$$
D^{\alpha} \bar{D}^{\beta} f(0)=0 \text { whenever } \sum_{j=1}^{n}\left(\alpha_{j}+\beta_{j}\right) \frac{1}{m_{j}} \leqslant \mu .
$$

If $n=1$ and $\Lambda=(1)$ then we use $\mathcal{O}(\mu)$ to denote the functions vanishing to order at least $\mu$ at the origin (cf. Cat84, Yu95]).

\section{Proofs of Theorem 1.1 and Theorem 1.2}

This section is devoted to our proofs of Theorem 1.1 and Theorem 1.2. First of all, let us recall the definition of the Kobayashi infinitesimal pseudometric and the Kobayashi pseudodistance as follows:

Definition 3.1. Let $M$ be a complex manifold. The Kobayashi infinitesimal pseudometric $F_{M}: M \times T^{1,0} M \rightarrow \mathbb{R}$ is defined by

$$
F_{M}(p, X)=\inf \left\{c>0 \mid \exists f: \Delta \rightarrow M \text { holomorphic with } f(0)=p, f^{\prime}(0)=X / c\right\},
$$

for any $p \in M$ and $X \in T^{1,0} M$, where $\Delta$ is the unit open disk of $\mathbb{C}$. Moreover, the Kobayashi pseudodistance $d_{M}^{K}: M \times M \rightarrow \mathbb{R}$ is defined by

$$
d_{M}^{K}(p, q)=\inf _{\gamma} \int_{0}^{1} F_{M}\left(\gamma(t), \gamma^{\prime}(t)\right) d t
$$

for any $p, q \in M$ where the infimum is taken over all differentiable curves $\gamma:[0,1] \rightarrow M$ joining $p$ and $q$. A complex manifold $M$ is called hyperbolic if $d_{M}^{K}(p, q)$ is actually a distance, i.e., $d_{M}^{K}(p, q)>0$ whenever $p \neq q$.

Next, we need the following lemma, whose proof will be given in Appendix for the convenience of the reader, and the following proposition.

Lemma 3.1. Assume that $\left\{D_{j}\right\}$ is a sequence of domains in $\mathbb{C}^{n+1}$ converging to a model $M_{P}$ of finite type. Then, we have

$$
\lim _{j \rightarrow \infty} F_{D_{j}}(z, X)=F_{M_{P}}(z, X), \forall(z, X) \in M_{P} \times \mathbb{C}^{n+1} .
$$

Moreover, the convergence takes place uniformly over compact subsets of $M_{P} \times \mathbb{C}^{n+1}$.

Proposition 3.2 (see [NN19]). Assume that $\left\{D_{j}\right\}$ is a sequence of domains in $\mathbb{C}^{n+1}$ converging to a model $M_{P}$ of finite type. Assume also that $\omega$ is a domain in $\mathbb{C}^{k}$ and $\sigma_{j}: \omega \rightarrow D_{j}$ is a sequence of holomorphic mappings such that $\left\{\sigma_{j}(a)\right\} \Subset M_{P}$ for some $a \in \omega$. Then $\left\{\sigma_{j}\right\}$ contains a subsequence that converges locally uniformly to a holomorphic map $\sigma: \omega \rightarrow M_{P}$.

Now we are ready to prove Theorem 1.1 and Theorem 1.2 . 
Proof of Theorem 1.1. Let $\rho$ be a local defining function for $\Omega$ near $\xi_{0}$ and the multitype $\mathcal{M}\left(\xi_{0}\right)=\left(1, m_{1}, \ldots, m_{n}\right)$ is finite. In what follows, denote by $\Lambda=\left(1 / m_{1}, \ldots, 1 / m_{n}\right)$. Since $\xi_{0}$ is a $h$-extendible point, there exist local holomorphic coordinates $(z, w)$ in which $\xi_{0}=0$ and $\Omega$ can be described in a neighborhood $U_{0}$ of 0 as follows:

$$
\Omega \cap U_{0}=\left\{\rho(z, w)=\operatorname{Re}(w)+P(z)+R_{1}(z)+R_{2}(\operatorname{Im} w)+(\operatorname{Im} w) R(z)<0\right\},
$$

where $P$ is a $\Lambda$-homogeneous plurisubharmonic real-valued polynomial containing no pluriharmonic terms, $R_{1} \in \mathcal{O}(1, \Lambda), R \in \mathcal{O}(1 / 2, \Lambda)$, and $R_{2} \in \mathcal{O}(2)$. (See the proof of Theorem 1.1 in [NN19] or the proof of Lemma 4.11 in YYu5].)

By assumption, there exists a point $a \in M$ such that the sequence $\eta_{j}:=f_{j}^{-1}(a)$ converges $\Lambda$-nontangentially to $\xi_{0}$. Without loss of generality, we may assume that the sequence $\left\{\eta_{j}\right\} \subset \Omega \cap U_{0}$ and we write $\eta_{j}=\left(\alpha_{j}, \beta_{j}\right)=\left(\alpha_{j 1}, \ldots, \alpha_{j n}, \beta_{j}\right)$ for all $j$. Then, the sequence $\left\{\eta_{j}:=f^{-1}(a)\right\}$ has the following properties:

(a) $\left|\operatorname{Im}\left(\beta_{j}\right)\right| \lesssim\left|\operatorname{dist}\left(\eta_{j}, \partial \Omega\right)\right|$;

(b) $\left|\alpha_{j k}\right|^{m_{k}} \lesssim\left|\operatorname{dist}\left(\eta_{j}, \partial \Omega\right)\right|$ for $1 \leqslant k \leqslant n$.

For the sequence $\left\{\eta_{j}=\left(\alpha_{j}, \beta_{j}\right)\right\}$, we associate with a sequence of points $\eta_{j}^{\prime}=$ $\left(\alpha_{j 1}, \ldots, \alpha_{j n}, \beta_{j}+\epsilon_{j}\right)$, where $\epsilon_{j}>0$, such that $\eta_{j}^{\prime}$ is in the hypersurface $\{\rho=0\}$ for all $j$. We note that $\epsilon_{j} \approx \operatorname{dist}\left(\eta_{j}, \partial \Omega\right)$. Now let us consider the sequences of dilations $\Delta^{\epsilon_{j}}$ and translations $L_{\eta_{j}^{\prime}}$, defined respectively by

$$
\Delta^{\epsilon_{j}}\left(z_{1}, \ldots, z_{n}, w\right)=\left(\frac{z_{1}}{\epsilon_{j}^{1 / m_{1}}}, \ldots, \frac{z_{n}}{\epsilon_{j}^{1 / m_{n}}}, \frac{w}{\epsilon_{j}}\right)
$$

and

$$
L_{\eta_{j}^{\prime}}(z, w)=(z, w)-\eta_{j}^{\prime}=\left(z-\alpha_{j}^{\prime}, w-\beta_{j}^{\prime}\right) .
$$

Under the change of variables $(\tilde{z}, \tilde{w}):=\Delta^{\epsilon_{j}} \circ L_{\eta_{j}^{\prime}}(z, w)$, i.e.,

$$
\left\{\begin{array}{l}
w-\beta_{j}^{\prime}=\epsilon_{j} \tilde{w} \\
z_{k}-\alpha_{j k}^{\prime}=\epsilon_{j}^{1 / m_{k}} \tilde{z}_{k}, k=1, \ldots, n,
\end{array}\right.
$$

one can see that $\Delta^{\epsilon_{j}} \circ L_{\eta_{j}^{\prime}}\left(\alpha_{j}, \beta_{j}\right)=(0, \cdots, 0,-1)$ for all $j$. Moreover, as in [NN19], after taking a subsequence if necessary, we may assume that the sequence of domains $\Omega_{j}:=\Delta^{\epsilon_{j}} \circ L_{\eta_{j}^{\prime}}\left(\Omega \cap U_{0}\right)$ converges to the following model

$$
M_{P, \alpha}:=\left\{(\tilde{z}, \tilde{w}) \in \mathbb{C}^{n} \times \mathbb{C}: \operatorname{Re}(\tilde{w})+P(\tilde{z}+\alpha)-P(\alpha)<0\right\},
$$

which is obviously biholomorphically equivalent to the model $M_{P}$. Without loss of generality, in what follows we always assume that $\left\{\Omega_{j}\right\}$ converges to $M_{P}$.

Now we first consider the sequence of biholomorphisms $F_{j}:=T_{j} \circ f_{j}^{-1}: M \supset f_{j}(\Omega \cap$ $\left.U_{0}\right) \rightarrow \Omega_{j}$, where $T_{j}:=\Delta^{\epsilon_{j}} \circ L_{\eta_{j}^{\prime}}$. Since $F_{j}(a)=\left(0^{\prime},-1\right)$ and notice that $f_{j}(\Omega \cap$ $U_{0}$ ) converges to $M$ as $j \rightarrow \infty$ (see Remark 2.2), by Proposition 3.2, without loss of generality, we may assume that the sequence $F_{j}$ converges uniformly on on every compact subset of $M$ to a holomorphic map $F$ from $M$ to $\mathbb{C}^{n+1}$. Note that $F(M)$ contains a neighborhood of $\left(0^{\prime},-1\right)$ and $F(M) \subset \overline{M_{P}}$.

Since $F_{j}$ is normal, by the Cauchy theorem it follows that $\left\{J\left(F_{j}\right)\right\}$ converges uniformly on every compact subsets of $M$ to $J(F)$, where $J(F)$ denotes the Jacobian determinant of $F$. However, by the Cartan theorem, $J\left(F_{j}\right)(z)$ is nowhere zero for any $j$ because $F_{j}$ is a biholomorphism. Then, the Hurwitz theorem implies that $J(F)$ is a zero function 
or nowhere zero. In the case that $J F \equiv 0, F$ is regular at no point of $M$. As $F(M)$ contains a neighborhood of $\left(0^{\prime},-1\right)$, the Sard theorem shows that $F$ is regular outside a proper subvariety of $M$, which is a contradiction. This yields $J F$ is nowhere zero and hence $F$ is regular everywhere on $M$. By [FS77, Lemma 0], it follows that $F(M)$ is open and $F(M) \subset M_{P}$.

Next, we shall prove that $F$ is one-to-one. Indeed, let $z_{1}, z_{2} \in M$ be arbitrary. Fix a compact subset $L \Subset M$ such that $z_{1}, z_{2} \in L$. Then, by Remark 2.2 there is a $j_{0}(L)>0$ such that $L \subset f_{j}\left(\Omega \cap U_{0}\right)$ and $F_{j}(L) \subset K \Subset M_{P}$ for all $j>j_{0}(L)$, where $K$ is a compact subset of $M_{P}$. By Lemma 3.1 and the decreasing property of Kobayashi distance, one has

$$
\begin{aligned}
d_{M}^{K}\left(z_{1}, z_{2}\right) & \left.\leqslant d_{f_{j}\left(\Omega \cap U_{0}\right)}^{K}\left(z_{1}, z_{2}\right)=d_{\Omega_{j}}^{K}\left(F_{j}\left(z_{1}\right), F_{j}\left(z_{2}\right)\right)\right) \leqslant C \cdot d_{M_{P}}^{K}\left(F_{j}\left(z_{1}\right), F_{j}\left(z_{2}\right)\right) \\
& \leqslant C\left(d_{M_{P}}^{K}\left(F\left(z_{1}\right), F\left(z_{2}\right)\right)+d_{M_{P}}^{K}\left(F_{j}\left(z_{1}\right), F\left(z_{1}\right)\right)+d_{M_{P}}^{K}\left(F_{j}\left(z_{2}\right), F\left(z_{2}\right)\right)\right),
\end{aligned}
$$

where $C>1$ is a positive constant. Letting $j \rightarrow \infty$, we obtain

$$
d_{M}^{K}\left(z_{1}, z_{2}\right) \leqslant C \cdot d_{M_{P}}^{K}\left(F\left(z_{1}\right), F\left(z_{2}\right)\right) .
$$

Since $M$ is hyperbolic, it follows that if $F\left(z_{1}\right)=F\left(z_{2}\right)$, then $z_{1}=z_{2}$. Consequently, $F$ is one-to-one, as desired.

Finally, because of the biholomorphism from $M$ to $F(M) \subset M_{P}$ and the tautness of $M_{P}$ (cf. [Yu95]), it follows that the sequence $F_{j}^{-1}=f_{j} \circ T_{j}^{-1}: T_{j}\left(\Omega \cap U_{0}\right) \rightarrow f_{j}\left(\Omega \cap U_{0}\right) \subset$ $M$ is also normal. Moreover, since $T_{j} \circ f_{j}^{-1}(a)=\left(0^{\prime},-1\right) \in M_{P}$, it follows that the sequence $T_{j} \circ f_{j}^{-1}$ is not compactly divergent. Therefore, by Proposition 2.1, after taking some subsequence we may assume that $T_{j} \circ f_{j}^{-1}$ converges uniformly on every compact subset of $M$ to a biholomorphism from $M$ onto $M_{P}$. Hence, the proof is complete.

Remark 3.3. If $M$ is a bounded domain in $\mathbb{C}^{n+1}$, the normality of the sequence $F_{j}^{-1}$ can be shown by using the Montel theorem. Thus, the proof of Theorem 1.1 simply follows from Proposition 2.1.

Proof of Theorem 1.2. Let $\rho$ be a local defining function for $\Omega$ near $\xi_{0}$. We may assume that $\xi_{0}=0$. After a linear change of coordinates, one can find local holomorphic coordinates $(\tilde{z}, \tilde{w})=\left(\tilde{z}_{1}, \cdots, \tilde{z}_{n}, \tilde{w}\right)$, defined on a neighborhood $U_{0}$ of $\xi_{0}$, such that

$$
\rho(\tilde{z}, \tilde{w})=\operatorname{Re}(\tilde{w})+\sum_{j=1}^{n}\left|\tilde{z}_{j}\right|^{2}+O\left(|\tilde{w}|\|\tilde{z}\|+\|\tilde{z}\|^{3}\right)
$$

By [DN09, Proposition 3.1] (or Subsection 3.1 in [Ber06] for the case $n=1$ ), for each point $\eta$ in a small neighborhood of the origin, there exists an automorphism $\Phi_{\eta}$ of $\mathbb{C}^{n}$ such that

$$
\rho\left(\Phi_{\eta}^{-1}(z, w)\right)-\rho(\eta)=\operatorname{Re}(w)+\sum_{j=1}^{n}\left|z_{j}\right|^{2}+O\left(|w|\|z\|+\|z\|^{3}\right) .
$$

Let us define an anisotropic dilation $\Delta^{\epsilon}$ by

$$
\Delta^{\epsilon}\left(z_{1}, \cdots, z_{n}, w\right)=\left(\frac{z_{1}}{\sqrt{\epsilon}}, \cdots, \frac{z_{n}}{\sqrt{\epsilon}}, \frac{w}{\epsilon}\right) .
$$


For each $\eta \in \partial \Omega$, if we set $\rho_{\eta}^{\epsilon}(z, w)=\epsilon^{-1} \rho \circ \Phi_{\eta}^{-1} \circ\left(\Delta^{\epsilon}\right)^{-1}(z, w)$, then

$$
\rho_{\eta}^{\epsilon}(z, w)=\operatorname{Re}(w)+\sum_{j=1}^{n}\left|z_{j}\right|^{2}+O(\sqrt{\epsilon}) .
$$

By assumption, the sequence $\eta_{j}:=f_{j}^{-1}(a)$ converges to $\xi_{0}$. Then, we associate with a sequence of points $\eta_{j}^{\prime}=\left(\eta_{j 1}, \cdots, \eta_{j n}, \eta_{j(n+1)}+\epsilon_{j}\right), \epsilon_{j}>0$, such that $\eta_{j}^{\prime}$ is in the hypersurface $\{\rho=0\}$. Then $\Delta^{\epsilon_{j}} \circ \Phi_{\eta^{\prime}}\left(\eta_{j}\right)=(0, \cdots, 0,-1)$ and one can see that $\Delta^{\epsilon_{j}} \circ \Phi_{\eta^{\prime}}(\{\rho=0\})$ is defined by an equation of the form

$$
\operatorname{Re}(w)+\sum_{j=1}^{n}\left|z_{j}\right|^{2}+O\left(\sqrt{\epsilon_{j}}\right)=0 .
$$

Therefore, it follows that, after taking a subsequence if necessary, $\Omega_{j}:=\Delta^{\epsilon_{j}} \circ \Phi_{\eta_{p}^{\prime}}\left(U_{0}^{-}\right)$ converges to the following domain

$$
\mathcal{E}:=\left\{\hat{\rho}:=\operatorname{Re}(w)+\sum_{j=1}^{n}\left|z_{j}\right|^{2}<0\right\},
$$

which is biholomorphically equivalent to the unit ball $\mathbb{B}^{n+1}$.

Now let us consider the sequence of biholomorphisms $F_{j}:=T_{j} \circ f_{j}^{-1}: M \supset f_{j}(\Omega \cap$ $\left.U_{0}\right) \rightarrow T_{j}\left(\Omega \cap U_{0}\right)$, where $T_{j}:=\Delta^{\epsilon_{j}} \circ \Phi_{\eta^{\prime}{ }_{j}}$. Since $F_{j}(a)=\left(0^{\prime},-1\right)$, by [DN09, Theorem 3.11], without loss of generality, we may assume that the sequence $F_{j}$ converges uniformly on every compact subset of $M$ to a holomorphic map $F$ from $M$ to $\mathbb{C}^{n+1}$. Note that $F(M)$ contains a neighborhood of $\left(0^{\prime},-1\right)$ and $F(M) \subset \overline{M_{P}}$. Following the argument as in the proof of Theorem 1.1, we conclude that $F$ is a biholomorphism from $M$ onto $\mathcal{E}$, and thus $M$ is biholomorphically equivalent to $\mathbb{B}^{n+1}$, as desired.

By Lemma 2.3 and Theorem [1.2, we obtain the following corollary, proved by F. S. Deng and X. J. Zhang [DZ19, Theorem 2.4] and by B. L. Fridman [Fr83, Theorem I].

Corollary 3.4. Let $D$ be a bounded strictly pseudoconvex domain in $\mathbb{C}^{n}$ with $\mathcal{C}^{2}$-smooth boundary. If a bounded domain $\Omega$ can be exhausted by $D$, then $\Omega$ is biholomorphically equivalent to $D$ or the unit ball $\mathbb{B}^{n}$.

\section{Exhausting a COMPLEX MANifold By A GENERAL ELLiPSOID}

In this section, we are going to prove that if a complex manifold $M$ can be exhausted by a general ellipsoid $D_{P}$ (see the definition of $D_{P}$ below), then $M$ is biholomorphically equivalent to either $D_{P}$ or the unit ball $B^{n}$.

First of all, let us fix $n$ positive integers $m_{1}, \ldots, m_{n-1}$ and denote by $\Lambda:=\left(\frac{1}{m_{1}}, \ldots, \frac{1}{m_{n-1}}\right)$. We assign weights $\frac{1}{m_{1}}, \ldots, \frac{1}{m_{n-1}}, 1$ to $z_{1}, \ldots, z_{n}$. For an $(n-1)$-tuple $K=\left(k_{1}, \ldots, k_{n-1}\right) \in$ $\mathbb{Z}_{\geqslant 0}^{n-1}$, denote the weight of $K$ by

$$
w t(K):=\sum_{j=1}^{k-1} \frac{k_{j}}{m_{j}} .
$$

Next, we consider the general ellipsoid $D_{P}$ in $\mathbb{C}^{n}(n \geqslant 2)$, defined by

$$
D_{P}:=\left\{\left(z^{\prime}, z_{n}\right) \in \mathbb{C}^{n}:\left|z_{n}\right|^{2}+P\left(z^{\prime}\right)<1\right\},
$$


where

$$
P\left(z^{\prime}\right)=\sum_{w t(K)=w t(L)=1 / 2} a_{K L} z^{\prime K} \bar{z}^{\prime L},
$$

where $a_{K L} \in \mathbb{C}$ with $a_{K L}=\bar{a}_{L K}$, satisfying that $P\left(z^{\prime}\right)>0$ whenever $z^{\prime} \in \mathbb{C}^{n-1} \backslash\left\{0^{\prime}\right\}$. We would like to emphasize here that the polynomial $P$ given in (2) is $\Lambda$-homogeneous and the assumption that $P\left(z^{\prime}\right)>0$ whenever $z^{\prime} \neq 0$ ensures that $D_{P}$ is bounded in $\mathbb{C}^{n}$ (cf. [NNTK19, Lemma 6]). Moreover, since $P\left(z^{\prime}\right)>0$ for $z^{\prime} \neq 0$ and by the $\Lambda$-homogeneity, there are two constants $c_{1}, c_{2}>0$ such that

$$
c_{1} \sigma_{\Lambda}\left(z^{\prime}\right) \leqslant P\left(z^{\prime}\right) \leqslant c_{2} \sigma_{\Lambda}\left(z^{\prime}\right), \forall z^{\prime} \in \mathbb{C}^{n-1},
$$

where $\sigma_{\Lambda}\left(z^{\prime}\right)=\left|z_{1}\right|^{m_{1}}+\cdots+\left|z_{n-1}\right|^{m_{n-1}}$. In addition, $D_{P}$ is called a WB-domain if it is strongly pseudoconvex at every boundary point outside the set $\left\{\left(0^{\prime}, e^{i \theta}\right): \theta \in \mathbb{R}\right\}$ (cf. AGK16]).

Now we prove the following proposition.

Proposition 4.1. Let $M$ be a n-dimensional complex hyperbolic manifold. Suppose that $M$ can be exhausted by the general ellipsoid $D_{P}$ via an exhausting sequence $\left\{f_{j}: D_{P} \rightarrow\right.$ $M\}$. If $D_{P}$ is a WB-domain, then $M$ is biholomorphically equivalent to either $D_{P}$ or the unit ball $\mathbb{B}^{n}$.

Remark 4.2. The possibility that $M$ is biholomorphic onto the unit ball $\mathbb{B}^{n}$ is not excluded because $D_{P}$ can exhaust the unit ball $\mathbb{B}^{n}$ by [FM95, Corollary 1.4].

Proof of Proposition 4.1. Let $q$ be an arbitrary point in $M$. Then, thanks to the boundedness of $D_{P}$, after passing to a subsequence if necessary we may assume that the sequence $\left\{f_{j}^{-1}(q)\right\}_{j=1}^{\infty}$ converges to a point $p \in \overline{D_{P}}$ as $j \rightarrow \infty$.

We now divide the argument into two cases as follows:

Case 1. $f_{j}^{-1}(q) \rightarrow p \in D_{P}$. Then, it follows from Lemma 2.3 that $M$ is biholomorphically equivalent to $D_{P}$.

Case 2. $f_{j}^{-1}(q) \rightarrow p \in \partial D_{P}$. Let us write $f_{j}^{-1}(q)=\left(a_{j}^{\prime}, a_{j n}\right) \in D_{P}$ and $p=\left(a^{\prime}, a_{n}\right) \in$ $\partial D_{P}$. As in [NNTK19, for each $j \in \mathbb{N}^{*}$ we consider $\psi_{j} \in \operatorname{Aut}\left(D_{P}\right)$, defined by

$$
\psi_{j}(z)=\left(\frac{\left(1-\left|a_{j n}\right|^{2}\right)^{1 / m_{1}}}{\left(1-\bar{a}_{j n} z_{n}\right)^{2 / m_{1}}} z_{1}, \ldots, \frac{\left(1-\left|a_{j n}\right|^{2}\right)^{1 / m_{n-1}}}{\left(1-\bar{a}_{j n} z_{n}\right)^{2 / m_{n-1}}} z_{n-1}, \frac{z_{n}-a_{j n}}{1-\bar{a}_{j n} z_{n}}\right) .
$$

Then $\psi_{j} \circ f_{j}(q)=\left(b_{j}, 0\right)$, where

$$
b_{j}=\left(\frac{a_{j 1}}{\left(1-\left|a_{j n}\right|^{2}\right)^{1 / m_{1}}}, \ldots, \frac{a_{j(n-1)}}{\left(1-\left|a_{j n}\right|^{2}\right)^{1 / m_{n-1}}}\right), \forall j \in \mathbb{N}^{*} .
$$

Without loss of generality, one may assume that $b_{j} \rightarrow b \in \mathbb{C}^{n-1}$ as $j \rightarrow \infty$.

Since $D_{P}$ is a $W B$-domain, two possibilities may occur:

Subcase 1: $p=\left(a^{\prime}, a_{n}\right)$ is a strongly pseudoconvex boundary point. In this subcase, it follows directly from Theorem 1.2 that $M$ is biholomorphically equivalent to $\mathbb{B}^{n}$.

Subcase 2: $p=\left(0^{\prime}, e^{i \theta}\right)$ is a weakly pseudoconvex boundary point. In this subcase, one must have $a_{j}^{\prime} \rightarrow 0^{\prime}$ and $a_{j n} \rightarrow e^{i \theta}$ as $j \rightarrow \infty$. Denote by $\rho(z):=\left|z_{n}\right|^{2}-1+P\left(z^{\prime}\right)$ a definition function for $D_{P}$. Then $\operatorname{dist}\left(a_{j}, \partial D_{P}\right) \approx-\rho\left(a_{j}\right)=1-\left|a_{j n}\right|^{2}-P\left(a_{j}^{\prime}\right)$. Suppose 
that $\left\{a_{j}\right\}$ converges $\Lambda$-nontangentially to $p$, i.e., $P\left(a_{j}^{\prime}\right) \approx \sigma_{\Lambda}\left(a_{j}^{\prime}\right) \lesssim \operatorname{dist}\left(a_{j}, \partial D_{P}\right)$, or equivalently $P\left(a_{j}^{\prime}\right) \leqslant C\left(1-\left|a_{j n}\right|^{2}-P\left(a_{j}^{\prime}\right)\right), \forall j \in \mathbb{N}^{*}$, for some $C>0$. This implies that

$$
P\left(a_{j}^{\prime}\right) \leqslant \frac{C}{1+C}\left(1-\left|a_{j n}\right|^{2}\right), \forall j \in \mathbb{N}^{*},
$$

and thus $P\left(b_{j}\right)=\frac{1}{1-\left|a_{j n}\right|^{2}} P\left(a_{j}^{\prime}\right) \leqslant \frac{C}{1+C}<1, \forall j \in \mathbb{N}^{*}$. This yields $\psi_{j} \circ f_{j}^{-1}(q)=$ $\left(b_{j}, 0\right) \rightarrow(b, 0) \in D_{P}$ as $j \rightarrow \infty$. So, again by Lemma 2.3 one concludes that $M$ is biholomorphically equivalent to $D_{P}$.

Now let us consider the case that the sequence $\left\{a_{j}\right\}$ does not converge $\Lambda$-nontangentially to $p$, i.e., $P\left(a_{j}^{\prime}\right) \geqslant c_{j} \operatorname{dist}\left(a_{j}, \partial D_{P}\right), \forall j \in \mathbb{N}^{*}$, where $0<c_{j} \rightarrow+\infty$. This implies that $P\left(a_{j}^{\prime}\right) \geqslant c_{j}^{\prime}\left(1-\left|a_{j n}\right|^{2}-P\left(a_{j}^{\prime}\right)\right), \forall j \in \mathbb{N}^{*}$, for some $0<c_{j}^{\prime} \rightarrow+\infty$, and hence

$$
P\left(a_{j}^{\prime}\right) \geqslant \frac{c_{j}^{\prime}}{1+c_{j}^{\prime}}\left(1-\left|a_{j n}\right|^{2}\right), \forall j \in \mathbb{N}^{*} .
$$

Thus, one obtains that $P\left(b_{j}\right)=\frac{1}{1-\left|a_{j n}\right|^{2}} P\left(a_{j}^{\prime}\right) \geqslant \frac{c_{j}^{\prime}}{1+c_{j}^{\prime}}$, which implies that $P(b)=$ 1. Consequently, $\psi_{j} \circ f_{j}^{-1}(q)$ converges to the strongly pseudoconvex boundary point $p^{\prime}=(b, 0)$ of $\partial D_{P}$. Hence, as in Subcase 1, it follows from Theorem 1.2 that $M$ is biholomorphically equivalent to $\mathbb{B}^{n}$.

Therefore, altogether, the proof of Proposition 4.1 finally follows.

\section{APPENDIX}

Proof of Lemma 3.1. We shall follow the proof of [Yu95, Theorem 2.1] with minor modifications. To do this, let us fix compact subsets $K \Subset M_{P}$ and $L \Subset \mathbb{C}^{n+1}$. Then it suffices to prove that $F_{D_{j}}(z, X)$ converges to $F_{M_{P}}(z, X)$ uniformly on $K \times L$. Indeed, suppose otherwise. Then, there exist $\epsilon_{0}>0$, a sequence of points $\left\{z_{j_{\ell}}\right\} \subset K$, and a sequence $X_{j_{\ell}} \subset L$ such that

$$
\left|F_{D_{j_{\ell}}}\left(z_{j_{\ell}}, X_{j_{\ell}}\right)-F_{M_{P}}\left(z_{j_{\ell}}, X_{j_{\ell}}\right)\right|>\epsilon_{0}, \forall \ell \geqslant 1
$$

By the homogeneity of the Kobayashi metrics $F(z, X)$ in $X$, we may assume that $\left\|X_{j_{\ell}}\right\|=1$ for all $\ell \geqslant 1$. Moreover, passing to subsequences, we may also assume that $z_{j_{\ell}} \rightarrow z_{0} \in K$ and $X_{j_{\ell}} \rightarrow X_{0} \in L$ as $\ell \rightarrow \infty$. Since $M_{P}$ is taut (see [Yu95, Theorem 3.13]), for each $(z, X) \in M_{P} \times \mathbb{C}^{n+1}$ with $X \neq 0$, there exists an analytic $\operatorname{disc} \varphi \in \operatorname{Hol}\left(\Delta, M_{P}\right)$ such that $\varphi(0)=z$ and $\varphi^{\prime}(0)=X / F_{M_{P}}(z, X)$. This implies that $F_{M_{P}}(z, X)$ is continuous on $M_{P} \times \mathbb{C}^{n+1}$. Hence, we obtain

$$
F_{M_{P}}\left(z_{j_{\ell}}, X_{j_{\ell}}\right) \rightarrow F_{M_{P}}\left(z_{0}, X_{0}\right)
$$

and thus we have

$$
\left|F_{D_{j_{\ell}}}\left(z_{j_{\ell}}, X_{j_{\ell}}\right)-F_{M_{P}}\left(z_{0}, X_{0}\right)\right|>\epsilon_{0} / 2
$$

for $\ell$ big enough.

By definition, for any $\delta \in(0,1)$ there exists a sequence of analytic discs $\varphi_{j_{\ell}} \in$ $\operatorname{Hol}\left(\Delta, D_{j_{\ell}}\right)$ such that $\varphi_{j_{\ell}}(0)=z_{0}, \varphi_{j_{\ell}}^{\prime}(0)=\lambda_{j_{\ell}} X_{j_{\ell}}$, where $\lambda_{j_{\ell}}>0$, and

$$
F_{D_{j_{\ell}}}\left(z_{j_{\ell}}, X_{j_{\ell}}\right) \geqslant \frac{1}{\lambda_{j_{\ell}}}-\delta
$$


It follows from Proposition 3.2 that every subsequence of the sequence $\left\{\varphi_{j_{\ell}}\right\}$ has a subsequence converging to some analytic disc $\psi \in \operatorname{Hol}\left(\Delta, M_{P}\right)$ such that $\psi(0)=$ $z_{0}, \psi^{\prime}(0)=\lambda X_{0}$, for some $\lambda>0$. Thus, one obtains that

$$
F_{M_{P}}\left(z_{0}, X_{0}\right) \leqslant \frac{1}{\left|\psi^{\prime}(0)\right|}
$$

for any such $\psi$. Therefore, one has

$$
\liminf _{\ell \rightarrow \infty} F_{D_{j_{\ell}}}\left(z_{j_{\ell}}, X_{j_{\ell}}\right) \geqslant F_{M_{P}}\left(z_{0}, X_{0}\right)-\delta
$$

On the other hand, as in [Yu95], by the tautness of $M_{P}$, there exists a analytic disc $\varphi \in \operatorname{Hol}\left(\Delta, M_{P}\right)$ such that $\varphi(0)=z_{0}, \varphi^{\prime}(0)=\lambda X_{0}$, where $\lambda=1 / F_{M_{P}}\left(z_{0}, X_{0}\right)$.

Now for $\delta \in(0,1)$, let us define an analytic disc $\psi_{j_{\ell}}^{\delta}: \Delta \rightarrow \mathbb{C}^{n+1}$ by settings:

$$
\psi_{j_{\ell}}^{\delta}(\zeta):=\varphi((1-\delta) \zeta)+\lambda(1-\delta)\left(X_{j_{\ell}}-X_{0}\right)+\left(z_{j_{\ell}}-z_{0}\right) \text { for all } \zeta \in \Delta .
$$

Since $\varphi((1-\delta) \bar{\Delta})$ is a compact subset of $M_{P}$ and $X_{j_{\ell}} \rightarrow X_{0}, z_{j \ell} \rightarrow z_{0}$ as $\ell \rightarrow \infty$, it follows that $\psi_{j_{\ell}}^{\delta}(\Delta) \subset D_{j_{\ell}}$ for all sufficiently large $\ell$, that is, $\psi_{j_{\ell}}^{\delta} \in \operatorname{Hol}\left(\Delta, D_{j_{\ell}}\right)$. Moreover, by construction, $\psi_{j_{\ell}}^{\delta}(0)=z_{j_{\ell}}$ and $\left(\psi_{j_{\ell}}^{\delta}\right)^{\prime}(0)=(1-\delta) \lambda X_{j_{\ell}}$. Therefore, again by definition, one has

$$
F_{D_{j_{\ell}}}\left(z_{j_{\ell}}, X_{j_{\ell}}\right) \leqslant \frac{1}{(1-\delta) \lambda}=\frac{1}{(1-\delta)} F_{M_{P}}\left(z_{0}, X_{0}\right)
$$

for all large $\ell$. Thus, letting $\delta \rightarrow 0^{+}$, one concludes that

$$
\limsup _{\ell \rightarrow \infty} F_{D_{j_{\ell}}}\left(z_{j_{\ell}}, X_{j_{\ell}}\right) \leqslant F_{M_{P}}\left(z_{0}, X_{0}\right) \text {. }
$$

By (41), (5), and (3), we seek a contradiction. Hence, the proof is complete.

Acknowledgement. Part of this work was done while the first author was visiting the Vietnam Institute for Advanced Study in Mathematics (VIASM). He would like to thank the VIASM for financial support and hospitality. The first author was supported by the Vietnam National Foundation for Science and Technology Development (NAFOSTED) under grant number 101.02-2017.311.

\section{REFERENCES}

[AGK16] T. Ahn, H. Gaussier, and K.-T. Kim, Positivity and completeness of invariant metrics, J. Geom. Anal. 26 (2) (2016), 1173-1185.

[Ber94] F. Berteloot, Characterization of models in $\mathbb{C}^{2}$ by their automorphism groups, Internat. J. Math., 5 (1994), 619-634.

[Ber06] F. Berteloot, Méthodes de changement d'échelles en analyse complexe, Ann. Fac. Sci. Toulouse Math. (6) 15 (2006), 427-483.

[Cat84] D. Catlin, Boundary invariants of pseudoconvex domains, Ann. of Math. (2) 120 (1984), no. $3,529-586$.

[DN09] Do Duc Thai and Ninh Van Thu, Characterization of domains in $\mathbb{C}^{n}$ by their noncompact automorphism groups, Nagoya Math. J. 196 (2009), 135-160.

[DT04] Do Duc Thai and Tran Hue Minh, Generalizations of the theorems of Cartan and GreeneKrantz to complex manifolds, Illinois J. of Math. 48 (2004), 1367-1384.

[DZ19] F. S. Deng and X. J. Zhang, Fridman's invariant, squeezing functions, and exhausting domains, Acta Math. Sin. (Engl. Ser.) 35 (2019), no. 10, 1723-1728. 
[For04] J. E. Fornæss, Short $\mathbb{C}^{k}$, in: Complex Analysis in Several Variables-Memorial Conference of Kiyoshi Oka's Centennial Birthday, in: Adv. Stud. Pure Math., vol. 42, Math. Soc. Japan, Tokyo, 2004, 95-108.

[FS77] J. E. Fornæss and E. L. Stout, Polydiscs in complex manifolds, Math. Ann. 227 (1977), no. 2, $145-153$.

[Fr83] B. L. Fridman, Biholomorphic invariants of a hyperbolic manifold and some applications, Trans. Amer. Math. Soc. 276 (1983), no. 2, 685-698.

[Fr86] B. L. Fridman, A universal exhausting domain, Proc. Amer. Math. Soc. 98 (1986), no. 2, 267270.

[FM95] B. L. Fridman and D. Ma, On exhaustion of domains, Indiana Univ. Math. J. 44 (1995), no. $2,385-395$.

[GK87] R. E. Greene and S.G. Krantz, Biholomorphic self-maps of domains, Lecture Notes in Math., 1276 (1987), 136-207.

[Liu18] B. Liu, Two applications of the Schwarz lemma, Pacific J. Math. 296 (2018), no. 1, 141-153.

[NN19] Ninh Van Thu and Nguyen Quang Dieu, Some properties of h-extendible domains in $\mathbb{C}^{n+1}, \mathrm{~J}$. Math. Anal. Appl. 485 (2020), no. 2, 123810, 14 pp..

[NNTK19] Ninh Van Thu, Nguyen Thi Lan Huong, Tran Quang Hung, and Hyeseon Kim, On the automorphism groups of finite multitype models in $\mathbb{C}^{n}$, J. Geom. Anal. 29 (2019), no. 1, 428-450.

[Yu94] J. Yu, Peak functions on weakly pseudoconvex domains, Indiana Univ. Math. J. 43 (1994), no. 4, 1271-1295.

[Yu95] J. Yu, Weighted boundary limits of the generalized Kobayashi-Royden metrics on weakly pseudoconvex domains, Trans. Amer. Math. Soc. 347(2) (1995), 587-614.

NinH VAN ThU

1 Department of Mathematics, Vietnam National University, Hanoi, 334 Nguyen Trai, Thanh Xuan, Hanoi, Vietnam

2 Thang Long Institute of Mathematics and Applied Sciences, Nghiem Xuan Yem, Hoang Mai, HaNoi, Vietnam

E-mail address: thunv@vnu.edu.vn

Trinh HuY Vu

1 Department of Mathematics, Vietnam National University at Hanoi, 334 Nguyen Trai Str., Hanoi, Vietnam

E-mail address: trinhhuyvu1508@gmail.com 